\title{
Procedimiento para la Selección de Criterios en la Planificación Energética de Zonas Rurales Colombianas
}

\author{
Carlos A. Robles-Algarín ${ }^{(1) \star}$, John A. Taborda-Giraldo ${ }^{(1)}$ y Adalberto J. Ospino-Castro(2) \\ (1) Universidad del Magdalena, Facultad de Ingeniería. Carrera 32 No. 22 - 08, Santa Marta-Colombia \\ (2) Universidad de la Costa, Facultad de Ingeniería. Calle 58 No. 55-66, Barranquilla-Colombia \\ (e-mail: carlosarturo.ing@gmail.com; jatabordag@gmail.com; aospino8@cuc.edu.co)
}

${ }^{*}$ Autor a quien debe ser dirigida la correspondencia

Recibido Oct. 5, 2017; Aceptado Nov. 14, 2017; Versión final Dic. 13, 2017, Publicado Jun. 2018

\begin{abstract}
Resumen
Este artículo presenta el procedimiento para la selección de un conjunto de criterios para tener en cuenta en proyectos de planificación energética para las zonas rurales de Colombia utilizando fuentes renovables de energía. La implementación de programas de electrificación en las zonas rurales colombianas se enfrenta a diversos obstáculos, entre los que se destaca la inadecuada selección de criterios para valorar la pertinencia de los proyectos. A partir de una revisión bibliográfica se identificaron 33 criterios que fueron sometidos al juicio de expertos con un cuestionario basado en la escala de Likert. Para la selección se utilizó como referente la media aritmética y el nivel de significancia. Se seleccionaron 20 subcriterios agrupados en 5 criterios: técnicos, económicos, sociales, ambientales y riesgo; los cuales permiten una evaluación integral de proyectos de planificación energética.
\end{abstract}

Palabras clave: planificación energética; electrificación rural; proyectos energéticos; fuentes renovables de energía; políticas energéticas

\section{A Procedure for Criteria Selection in the Energy Planning of Colombian Rural Areas}

\begin{abstract}
This paper presents the process of selecting a set of criteria for use in energy planning processes using renewable energy sources in rural areas of Colombia. The implementation of renewable energies in the electrification programs of rural areas in Colombia has several obstacles, among which the inadequate selection of criteria to assess the relevance of projects is highlighted. From a bibliographic review, 33 criteria were identified and submitted to expert judgment with a questionnaire based on the Likert scale. For the selection process, the arithmetic mean and the level of significance were used as reference. After processing the results, 20 subcriteria were selected and grouped into five criteria: technical, economic, social, environmental and risk; which allow a comprehensive evaluation of energy planning projects.
\end{abstract}

Keywords: energy planning; rural electrification; energy projects; renewable energy sources; energy policies 


\section{INTRODUCCIÓN}

En la actual era del conocimiento y de la aplicación de las tecnologías de la información, es difícil imaginar cuantas de las actividades del día a día se pueden realizar sin el uso de la energía. Lamentablemente a nivel mundial no existe una cultura generalizada sobre la importancia que tiene el uso eficiente de la energía. En la medida que una sociedad es más desarrollada consume más energía y en la mayoría de los casos de forma ineficiente. Por lo tanto, se hace necesario establecer un desarrollo socioeconómico compatible con un modelo energético sostenible que proteja el medio ambiente y pueda generar bienestar a las comunidades haciendo uso de recursos renovables. (Ahmad y Tahar, 2014; Emodi et al., 2015)

Según el más reciente reporte global de la Agencia Internacional de las Energías Renovables (IRENA), en el 2014 el 19.2\% de la energía primaria consumida en el mundo fue producida a partir de energías renovables (ER). Se destaca en primer lugar el conjunto de ER modernas con el $10.3 \%$ y posteriormente la biomasa tradicional con el $8.9 \%$ (IRENA, 2016). Para el caso de las zonas rurales, el potencial de las ER está creciendo aceleradamente debido a la disminución de los precios, las aplicaciones en sectores como la agricultura, educación y salud (Mizanur et al., 2013). No obstante, en la mayoría de las zonas rurales de los países en vía de desarrollo, las necesidades de energía se satisfacen principalmente con combustibles derivados de la biomasa. Este panorama limita seriamente la posibilidad de muchos pobladores de las zonas rurales de mejorar la productividad agrícola y su calidad de vida.

En el caso colombiano, según la Unidad de Planeación Minero Energética (UPME), en gran parte de las zonas rurales se cuenta con el potencial para construir soluciones para el suministro de energía en los campos de la biomasa, fotovoltaica, eólica y microcentrales; especialmente si se dan por hecho los altos costos de instalación, operación y mantenimiento de la red convencional para las zonas apartadas. En estas zonas los proyectos se concentran en aplicaciones térmicas para calentamiento de agua en sectores residenciales, electrificación rural y suministro energético en equipos de telecomunicaciones (UPME, 2015).

Teniendo en cuenta lo anterior, la UPME a través de su plan de desarrollo 2011-2020 para las Fuentes no Convencionales de Energía (FNCE) realizado por el Consorcio Energético CORPOEMA, ha establecido como un subprograma estratégico la promoción del uso de FNCE; para lo cual el plan adopta que para el año 2020 las FNCE tengan $6.5 \%$ de participación en el Sistema Interconectado Nacional (SIN) y $30 \%$ en las Zonas No Interconectadas (ZNI). Aproximadamente el $78 \%$ de la energía que se consume en Colombia proviene de recursos fósiles y el $22 \%$ restante proviene de energías renovables. En el SIN se cuenta con una capacidad instalada en ER de $15645 \mathrm{MW}$, donde el mayor porcentaje de participación lo tienen las hidroeléctricas mayores a $20 \mathrm{MW}$ con el $66.6 \%$. La menor participación es para las plantas de cogeneración de biomasa con el $0.5 \%$ y para las plantas eólicas con el $0.1 \%$. Para el caso de las ZNI se tiene una capacidad instalada de 167MW distribuidas en 1269 plantas de generación (Corpoema, 2010). Sin embargo, la aplicación de las ER en las zonas rurales colombianas se enfrenta a varios problemas. Generalmente las entidades que promocionan los proyectos energéticos en las zonas rurales están a cargo de organizaciones no gubernamentales, así como de gobiernos regionales, los cuales al no contar con los recursos económicos y personal altamente cualificado suelen replicar proyectos estandarizados que no necesariamente se adaptan a todas las zonas.

Históricamente se han fijado estrategias vagas para promover el uso de las ER, las cuales se centran en la aplicación de subsidios para la electrificación en las zonas rurales. No hay claridad en los criterios para valorar la pertinencia social y ambiental de los proyectos, así como políticas orientadas a la adquisición del conocimiento local sobre ER con mayor perspectiva de desarrollo en el país (Garcia y Zerda, 2016). Esta situación contrasta con la de los países desarrollados en los cuales se fijan políticas en ER ligadas a metas de reducción de emisiones y disminución de la dependencia del suministro externo (Amer y Daim, 2011).

Dentro de este marco, según se presenta en Rojas (2015), en la literatura se documentan diversas experiencias que demuestran lo inconveniente de realizar el tipo de prácticas anteriormente mencionadas. Se destaca el inconformismo por parte de los usuarios al ver que no son cubiertas sus necesidades básicas. En muchos casos se realizan sistemas sobredimensionados que hacen que los usuarios asuman extra costos por la utilización que hacen de la energía. Asimismo, se realizan proyectos de generación que priorizan los criterios técnico-económicos dejando de lado los aspectos sociales, ambientales y de riesgo que garanticen la participación de las comunidades locales con mayor perspectiva en el desarrollo del país. Además, se presentan problemas con las comunidades que no generan los recursos económicos necesarios para suplir costos de mantenimiento y reposición de equipos. Por ser comunidades aisladas es necesario que exista un conocimiento local que esté capacitado para realizar tareas de mantenimiento preventivo, actualización y reparación de equipos. 
Todo lo anteriormente expuesto refleja que existen muchas debilidades en los proyectos de electrificación rural que ocasionan inconformismo en los usuarios limitando sus perspectivas de desarrollo. Estas situaciones generan desconfianza en las nuevas tecnologías condicionando la aplicación de futuros proyectos. Por tal motivo, recientemente se están trabajando proyectos que promueven la electrificación rural haciendo uso de fuentes renovables de energía teniendo en cuenta múltiples aspectos, lo cual permite incorporar en la planificación energética diferentes criterios como los técnicos, económicos, sociales y ambientales. (Demirtas, 2013; Kumar et al., 2016; Rojas y Yusta, 2015; Aragonés et al., 2014; Espejo et al., 2017; Robles et al., 2017; Şengül et al., 2015).

En ese sentido, en el presente artículo se presenta un procedimiento para la selección de criterios en proyectos de planificación energética para las zonas rurales colombianas. Inicialmente se presenta la metodología utilizada, en la cual se utilizaron 33 subcriterios preliminares que fueron sometidos a la valoración de expertos. Se definió un baremo que permitió establecer el nivel de significancia de cada uno de los subcriterios. Posteriormente se presentan los resultados, los cuales reflejan que a juicio de los expertos se deben tener en cuenta 20 subcriterios distribuidos en los criterios técnicos, económicos, sociales, ambientales y riesgo.

\section{METODOLOGÍA}

El propósito de este estudio consiste en definir los criterios a tener en cuenta para proyectos de planificación energética con énfasis en las zonas rurales colombianas. De esta forma, se realizó una investigación descriptiva con un diseño de campo que permitió acudir a la valoración de expertos para la selección de los criterios. A continuación, se presenta el procedimiento implementado para la definición de los criterios.

\section{Criterios preliminares}

La primera actividad consistió en realizar una revisión bibliográfica de investigaciones a nivel nacional e internacional en el área de planificación energética para establecer un conjunto de criterios preliminares que posteriormente fueron sometidos a la valoración de expertos. De esta forma, se elaboró una lista preliminar de 33 criterios teniendo en cuenta los autores consultados y la pertinencia inicial para utilizarlos en proyectos de planificación energética en el contexto colombiano. En la Tabla 1 se muestran los criterios establecidos con los principales autores consultados.

\section{Selección de criterios}

Para la selección de los criterios se realizó la consulta a un grupo de expertos distribuidos de la siguiente forma: 1) Empresas del sector de las fuentes renovables de energía; 2) Profesores universitarios investigadores con experiencia en proyectos de planificación energética; 3) Empleados públicos con funciones asociadas al uso racional de los recursos y proyectos de planificación energética; 4) Organizaciones no gubernamentales protectoras del medio ambiente; y 5) organizaciones pertenecientes a las comunidades rurales.

Tabla 1: Criterios preliminares.

\begin{tabular}{|l|l|}
\hline Criterio & Referente Principal \\
\hline Madurez de la tecnología: etapa de desarrollo de la energía renovable a utilizar. & (Demirtas, 2013) \\
\hline $\begin{array}{l}\text { Inversión inicial: costo total de equipos y materiales necesarios para realizar la } \\
\text { instalación del sistema renovable. }\end{array}$ & (Kumar et al., 2016) \\
\hline $\begin{array}{l}\text { Conflicto armado: riesgos de seguridad relacionados con el conflicto armado que se } \\
\text { vive en Colombia. }\end{array}$ & (Gülçin y Sezin, 2014) \\
\hline $\begin{array}{l}\text { Instalación en territorios indígenas: aceptación de los indígenas respecto a la la } \\
\text { instalación del sistema renovable en territorios considerados sagrados. }\end{array}$ & (Rosso et al., 2014) \\
\hline $\begin{array}{l}\text { Fallas en equipos: vulnerabilidad de los equipos que le puedan ocasionar fallas en } \\
\text { su funcionamiento. }\end{array}$ & (Guerrero et al., 2016) \\
\hline $\begin{array}{l}\text { Impacto en el ecosistema: impacto negativo que tendría el sistema renovable sobre } \\
\text { las especies locales. }\end{array}$ & (Kahraman et al., 2009) \\
\hline $\begin{array}{l}\text { Salarios: monto de los salarios que se pagaría a los miembros de la comunidad que } \\
\text { trabajen en la implementación y mantenimiento del sistema energético renovable. }\end{array}$ & (Mourmouris y Potolias, 2013) \\
\hline Eficiencia energética: eficiencia de conversión del sistema renovable. & (Shabbar et al., 2014) \\
\hline $\begin{array}{l}\text { Energía para escuelas y centros de salud: capacidad del sistema para suministrar } \\
\text { energía eléctrica en las escuelas y centros de salud de la comunidad. }\end{array}$ & (Robles et al., 2017) \\
\hline
\end{tabular}


Tabla 1 (continuación)

\begin{tabular}{|c|c|}
\hline $\begin{array}{l}\text { Obsolescencia de equipos: Equipos que se dejen de fabricar y no puedan ser } \\
\text { reemplazados en casos de averías. }\end{array}$ & (Tasri y Susilawati, 2014) \\
\hline Impacto en el entorno: impacto luego de la implementación del sistema. & (Mizanur et al., 2013) \\
\hline Costos imprevistos: gastos imprevistos que se generan durante la implementación. & (Zanuttigh et al., 2016) \\
\hline $\begin{array}{l}\text { Riesgos del país: riesgo asociado a la corrupción, inestabilidad política y económica } \\
\text { del país. }\end{array}$ & (UPME, 2015) \\
\hline $\begin{array}{l}\text { Acceso de la tecnología: distribución igualitaria de los servicios de energía para todos } \\
\text { los miembros de la comunidad. }\end{array}$ & (Rojas y Yusta, 2015) \\
\hline $\begin{array}{l}\text { Fenómenos naturales: riesgos asociados a la ocurrencia de fenómenos naturales } \\
\text { como tormentas, fuertes lluvias, terremotos e inundaciones. }\end{array}$ & (Aragonés et al., 2014) \\
\hline $\begin{array}{l}\text { Política energética: riesgo de cambios en la política energética nacional que puedan } \\
\text { afectar la viabilidad económica del sistema renovable. }\end{array}$ & (Gülçin y Sezin, 2014) \\
\hline $\begin{array}{l}\text { Disponibilidad de repuestos y mantenimiento: disponibilidad local de repuestos para } \\
\text { actividades de mantenimiento preventivo y correctivo en pequeñas y grandes } \\
\text { averías. }\end{array}$ & (Hernández et al., 2015) \\
\hline Fácil acceso: existencia de vías para un acceso fácil y seguro a las instalaciones. & (Corpoema, 2010) \\
\hline Confiabilidad: capacidad del sistema para funcionar según las condiciones de diseño. & (Baris y Kucukali, 2012) \\
\hline $\begin{array}{l}\text { Costos de operación y mantenimiento: costos de mantenimiento preventivo, } \\
\text { correctivo y mano de obra utilizada. }\end{array}$ & (Kang et al., 2011) \\
\hline $\begin{array}{l}\text { Horas de operación continua: autonomía del sistema renovable para suministrar } \\
\text { energía sin interrupciones. }\end{array}$ & (Ahmad y Tahar, 2014) \\
\hline $\begin{array}{l}\text { Generación de empleos locales: número de empleos locales que se generarían en } \\
\text { actividades de instalación, mantenimiento y reparación del sistema renovable. }\end{array}$ & (Demirtas, 2013) \\
\hline $\begin{array}{l}\text { Emisiones de gases: emisiones de gases de efecto invernadero que produzca el } \\
\text { sistema energético renovable. }\end{array}$ & (Zhang et al., 2012) \\
\hline $\begin{array}{l}\text { Retorno de la inversión: Tiempo necesario para recuperar la inversión inicial } \\
\text { realizada. }\end{array}$ & (Kuthanazhi y Rao, 2012) \\
\hline $\begin{array}{l}\text { Producción de residuos: generación de residuos que impacten el ambiente y la } \\
\text { comunidad. }\end{array}$ & (Tasri y Susilawati, 2014) \\
\hline $\begin{array}{l}\text { Infraestructura física: disponibilidad de infraestructura física que garantice la } \\
\text { implementación y puesta en marcha del sistema energético renovable. }\end{array}$ & (Amer y Daim, 2011) \\
\hline $\begin{array}{l}\text { Incentivos para la inversión: incentivos legales existentes que se obtendrían al } \\
\text { implementar el sistema renovable. }\end{array}$ & (Zanuttigh et al., 2016) \\
\hline $\begin{array}{l}\text { Aceptación de la comunidad: disposición de la comunidad para aceptar la } \\
\text { implementación del sistema renovable en sus localidades. }\end{array}$ & (Rojas y Yusta, 2015) \\
\hline Vida útil: vida útil del sistema renovable garantizando un correcto funcionamiento. & (Mourmouris y Potolias, 2013) \\
\hline $\begin{array}{l}\text { Recursos locales (agua y suelo): superficie del suelo y recursos hídricos necesarios } \\
\text { para implementar el sistema renovable. }\end{array}$ & (Ahmad y Tahar, 2014) \\
\hline $\begin{array}{l}\text { Impacto acústico: ruido debido a la implementación del sistema renovable y que } \\
\text { podría afectar a los miembros de la comunidad. }\end{array}$ & (Kahraman et al., 2009) \\
\hline $\begin{array}{l}\text { Tiempo de espera: tiempo transcurrido desde la construcción a la operación del } \\
\text { sistema. }\end{array}$ & (Parodi, 2013) \\
\hline $\begin{array}{l}\text { Potencial de recursos: disponibilidad de los recursos naturales para la generación } \\
\text { con fuentes renovable de energía. }\end{array}$ & (UPME, 2015) \\
\hline
\end{tabular}

Se elaboró un cuestionario que fue respondido 47 expertos con el fin de identificar los criterios a tener en cuenta en proyectos de planificación energética en las zonas rurales colombianas. El cuestionario estuvo integrado por 33 preguntas y se definieron las siguientes categorías de acuerdo a la escala de Likert con una escala de 1 a 5 de la siguiente forma: (5) Totalmente de acuerdo, (4) De acuerdo, (3) Ni de acuerdo, ni en desacuerdo, (2) En desacuerdo y (1) Totalmente en desacuerdo. La escala Likert permite establecer el nivel de acuerdo o desacuerdo de un experto respecto a un enunciado (Gosavi, 2015; Simanca et al., 2016). El cuestionario fue elaborado utilizando un formulario de Google y fue enviado vía correo electrónico a cada uno de los expertos. Para cada criterio se calculó la media y la desviación estándar a partir de las respuestas de los expertos en relación a los criterios. Adicionalmente, se diseñó un baremo y se establecieron 5 atributos relacionados con el nivel de significancia, como se puede ver en la Tabla 2. Los atributos señalan la pertinencia de incluir o no los criterios en el proceso de planificación energética. La escala para la evaluación de cada uno de los criterios fue elaborada a partir de la ecuación (1):

$$
\mathrm{R}=\left(\mathrm{L}_{\mathrm{s}}-\mathrm{L}_{\mathrm{i}}\right) / \mathrm{n}
$$


En esta ecuación, $R$ es el rango que se utiliza en cada intervalo, $L_{s}$ es el límite superior de la escala utilizada, $\mathrm{L}_{i}$ es el límite inferior, y $\mathrm{n}$ es el número de atributos del baremo. De esta forma, la amplitud para cada rango definido es de (5-1)/5, es decir 0.8. Por lo tanto, el baremo quedó establecido según se muestra en la Tabla 2. Para la selección final se establecieron 5 criterios: técnicos, económicos, sociales, ambientales y de riesgo. Posteriormente se seleccionaron los subcriterios cuya media individual superara la media del criterio al que fue asignado. Además, de acuerdo al baremo, se utilizó como indicador de selección que cada criterio tuviera un nivel de significancia Muy alto o Alto.

Tabla 2: Baremo para la selección de los criterios.

\begin{tabular}{|l|l|l|}
\hline Categorías & Rango de Valores & Atributos \\
\hline Totalmente de acuerdo & $4,2 \leq \mathrm{R} \leq 5,0$ & Muy alta significancia \\
\hline De acuerdo & $3,4 \leq \mathrm{R}<4,2$ & Alta significancia \\
\hline $\begin{array}{l}\text { Ni de acuerdo, ni en } \\
\text { desacuerdo }\end{array}$ & $2,6 \leq \mathrm{R}<3,4$ & Moderada significancia \\
\hline En desacuerdo & $1,8 \leq \mathrm{R}<2,6$ & Poca significancia \\
\hline Totalmente en desacuerdo & $1 \leq \mathrm{R}<1,8$ & Muy poca significancia \\
\hline
\end{tabular}

\section{Confiabilidad}

Para medir la confiabilidad del instrumento se utilizó el Alfa de Cronbach, el cual es uno de los métodos de consistencia más ampliamente utilizado por los investigadores para estimar la fiabilidad de cuestionarios, en los casos que se utilizan conjunto de ítems que buscan medir el mismo atributo o campo de contenido como es el caso de la presente investigación. Se resalta como principal ventaja que sólo se requiere la aplicación de una prueba y su facilidad de cómputo. Se calculó de acuerdo a la ecuación (2) (De la Hoz et al., 2016).

$$
\propto=\left(\frac{n}{n-1}\right)\left(1-\frac{\sum_{i=1}^{n} \sigma_{i}^{2}}{\sigma_{x}^{2}}\right)
$$

Donde $n$ es el número de ítems de la prueba, $\sigma_{i}^{2}$ corresponde a la varianza de los ítems desde 1 hasta i y $\sigma_{x}^{2}$ es la varianza total de la prueba. El coeficiente permite medir la confiabilidad del cuestionario en función de dos términos: el número de los ítems y la proporción de la varianza total debido a la covarianza entre los ítems.

En atención a todo lo anteriormente expuesto, para estimar la confiabilidad del instrumento se aplicó una prueba piloto a un grupo de expertos diferente a la población de estudio, pero con las mismas características que se abordaron en la presente investigación. De esta forma, para calcular la confiabilidad se utilizó la ecuación (2) del Alfa de Cronbach con los siguientes datos: i) Número de ítems: 33; ii) Suma de la varianza de cada ítem: 62.22; y iii) Varianza total de la prueba: 275.15. Con estos valores se obtuvo un Alfa de Cronbach de 0.80 , el cual equivale a un valor de confiablidad aceptable según lo establecido por (Hernández et al., 2010).

\section{RESULTADOS}

En la Tabla 3 se muestran la media, desviación estándar y nivel de significancia que se obtuvo para cada uno de los subcriterios luego de analizar los resultados del instrumento aplicado al grupo de expertos. Se puede observar que el mayor valor promedio fue obtenido por la disponibilidad de recursos locales (agua y suelo) con una media de 4.55 y el menor valor fue para el tiempo de espera con una media de 1.94. De igual forma, se observa que la desviación estándar que presentan los criterios respecto a la media oscila entre 0.72 y 1.60 ; lo cual refleja el nivel de dispersión de los juicios emitidos por los expertos.

A partir de los resultados obtenidos, según se detalló en la metodología, se establecieron 5 criterios: técnicos, económicos, sociales, ambientales y riesgo. Para los criterios técnicos, se agruparon los siguientes subcriterios: madurez de la tecnología (C1.1), eficiencia energética (C1.2), disponibilidad de repuestos y mantenimiento (C1.3), confiabilidad (C1.4), horas de operación continua (C1.5), infraestructura física (C1.6) y tiempo de espera (C1.7).

Para realizar la selección de los subcriterios técnicos, se realizó un análisis de acuerdo a lo presentado en la figura 1, donde se seleccionaron los subcriterios cuya media individual superara a la media grupal. Además, los subcriterios seleccionados cumplen con un nivel de significancia Alto. Por consiguiente, se seleccionaron 5 subcriterios técnicos: C1.1, C1.2, C1.3, C1.4 y C1.6. Con este análisis quedaron establecidos los criterios técnicos, con una media de 3.21 que equivale a un nivel Moderado de significancia. 
Tabla 3: Resultados obtenidos para los subcriterios.

\begin{tabular}{|c|c|c|c|}
\hline Subcriterios & Media & $\begin{array}{l}\text { Desviación } \\
\text { Estándar }\end{array}$ & $\begin{array}{l}\text { Significancia } \\
\text { Según Baremo }\end{array}$ \\
\hline Madurez de la tecnología & 4.17 & 0.79 & Alta \\
\hline Inversión inicial & 3.74 & 1.33 & Alta \\
\hline Conflicto armado & 2.68 & 1.30 & Moderada \\
\hline Instalación en territorios indígenas & 3.64 & 1.26 & Alta \\
\hline Fallas en equipos & 2.23 & 1.39 & Poca \\
\hline Impacto en el ecosistema & 4.23 & 1.22 & Alta \\
\hline Salarios & 2.21 & 1.14 & Poca \\
\hline Eficiencia energética & 3.77 & 1.24 & Alta \\
\hline Energía para escuelas y centros de salud & 3.91 & 1.32 & Alta \\
\hline Obsolescencia de equipos & 2.32 & 1.39 & Poca \\
\hline Impacto en el entorno & 2.68 & 1.30 & Moderada \\
\hline Costos imprevistos & 2.77 & 1.32 & Moderada \\
\hline Riesgos del país & 3.53 & 1.28 & Alta \\
\hline Acceso de la tecnología & 2.13 & 1.13 & Poca \\
\hline Fenómenos naturales & 3.68 & 1.38 & Alta \\
\hline Política energética & 3.77 & 1.49 & Alta \\
\hline Disponibilidad de repuestos y mantenimiento & 3.64 & 1.45 & Alta \\
\hline Fácil acceso & 3.66 & 1.32 & Alta \\
\hline Confiabilidad & 3.53 & 1.52 & Alta \\
\hline Costos de operación y mantenimiento & 3.64 & 1.45 & Alta \\
\hline Horas de operación continua & 2.17 & 1.32 & Poca \\
\hline Generación de empleos locales & 4.26 & 1.17 & Muy Alta \\
\hline Emisiones de gases & 3.55 & 1.47 & Alta \\
\hline Retorno de la inversión & 3.11 & 1.60 & Moderada \\
\hline Producción de residuos & 3.45 & 1.27 & Alta \\
\hline Infraestructura física & 3.49 & 1.41 & Alta \\
\hline Incentivos para la inversión & 3.53 & 1.46 & Alta \\
\hline Aceptación de la comunidad & 4.53 & 0.88 & Muy Alta \\
\hline Vida útil & 3.23 & 1.42 & Moderada \\
\hline Recursos locales (agua y suelo) & 4.55 & 0.72 & Muy Alta \\
\hline Impacto acústico & 2.74 & 1.44 & Moderada \\
\hline Tiempo de espera & 1.94 & 1.28 & Poca \\
\hline Potencial de recursos & 3.83 & 1.42 & Alta \\
\hline
\end{tabular}

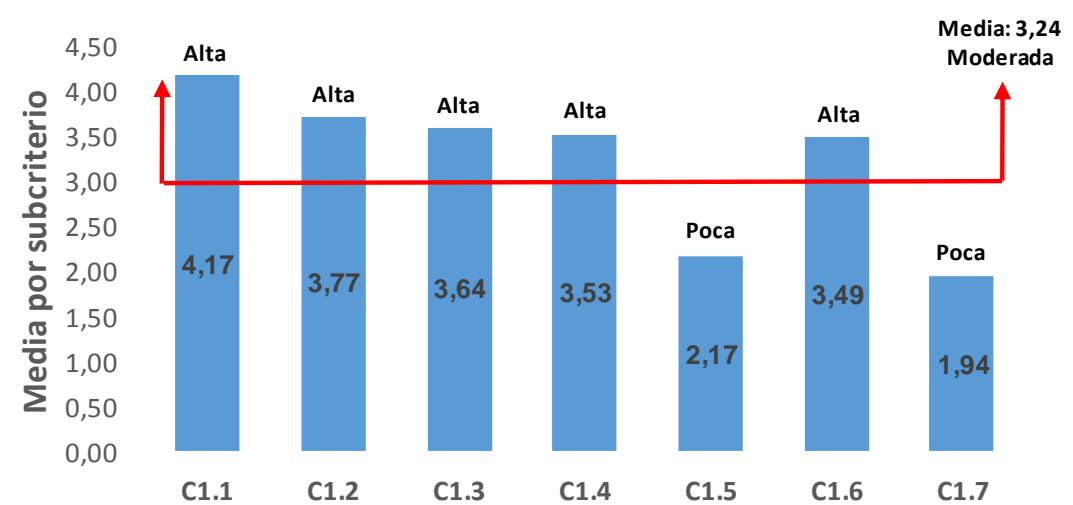

Subcriterios Técnicos

Fig. 1: Selección de subcriterios técnicos.

En el caso de los criterios económicos se agruparon los siguientes subcriterios: inversión inicial (C2.1), costos imprevistos (C2.2), costos de operación y mantenimiento (C2.3), retorno de la inversión (C2.4), incentivos para la inversión (C2.5), vida útil (C2.6) y potencial de recursos (C2.7). De acuerdo con la información presentada en la figura 2, se seleccionaron los subcriterios C2.1, C2.3, C2.5 y C2.7, los cuales superaron la media grupal y tienen un nivel de significancia Alto. De esta forma, quedaron definidos los subcriterios económicos con una media de 3.44 y un Alto nivel de significancia. Para los criterios sociales se agruparon los siguientes subcriterios: instalación en territorios indígenas (C3.1), salarios (C3.2), energía para escuelas y centros de salud (C3.3), acceso a la tecnología (C3.4), generación de empleos locales (C3.5) y aceptación 
de la comunidad (C3.6). Teniendo en cuenta la figura 3, se seleccionaron los subcriterios C3.1, C3.3, C3.5 y C3.6, los cuales tuvieron nivel de significancia Alto o Muy Alto y superaron la media del grupo. Con este procedimiento quedaron definidos los subcriterios sociales con una media de 3.45 y un nivel de significancia Alto.



Fig. 2: Selección de subcriterios económicos.

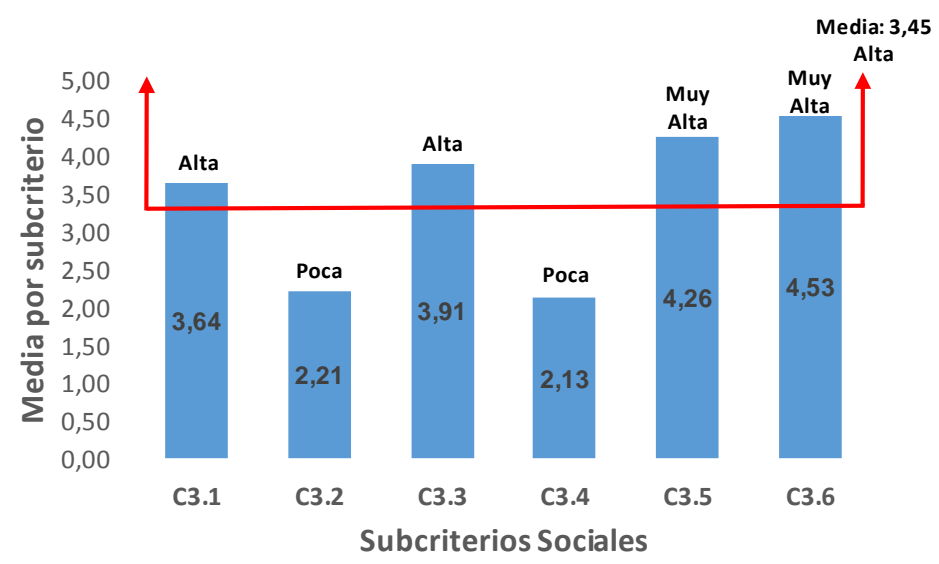

Fig. 3: Selección de subcriterios sociales.

En el caso de los criterios ambientales, se agruparon los siguientes subcriterios: impacto en el entorno (C4.1), impacto en el ecosistema (C4.2), emisiones de gases (C4.3), producción de residuos (C4.4), recursos locales: agua y suelo (C4.5) e impacto acústico (C4.6). A partir de la figura 4, se seleccionaron los subcriterios C4.2, C4.3 y C4.6, los cuales superan la media grupal y además tienen niveles de significancia Alto y Muy Alto. Es de anotar que los criterios ambientales tuvieron una media de 3.54 con un nivel de significancia Alto.

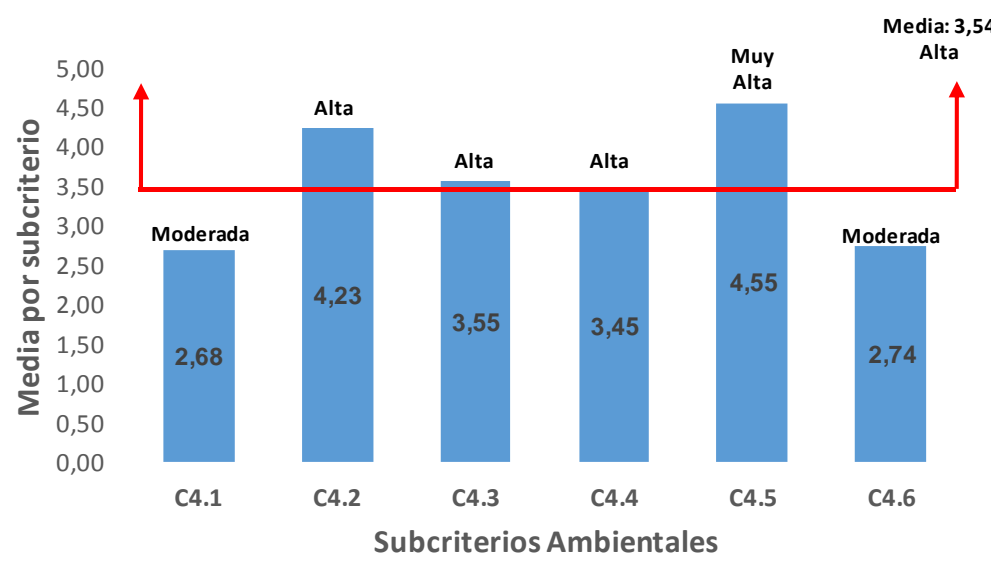

Fig. 4: Selección de subcriterios ambientales. 
Finalmente, para los criterios de riesgo se agruparon los siguientes subcriterios: conflicto armado (C5.1), fallas en equipos (C5.2), obsolescencia de equipos (C5.3), riesgos del país (C5.4), fenómenos naturales (C5.5), política energética (C5.6) y fácil acceso (C5.7). De acuerdo a los resultados mostrados en la figura 5, se seleccionaron los subcriterios C5.4, C5.5, C5.6 y C5.7, los cuales superaron la media grupal y tienen un Alto nivel de significancia. Con este análisis, los criterios de riesgo tuvieron un nivel de significancia Moderado con una media de 3.12 .



Fig. 5: Selección de subcriterios de riesgo.

Según el análisis realizado a partir de los resultados obtenidos con la valoración de los expertos, en la Tabla 4 se resumen los subcriterios seleccionados para proyectos de planificación energética con fuentes renovables de energías en las zonas rurales colombianas. En total se seleccionaron 20 subcriterios, los cuales fueron ordenados de mayor a menor de acuerdo a la media que obtuvieron dentro de la categoría.

Tabla 4: Subcriterios seleccionados.

\begin{tabular}{|l|l|l|l|l|}
\hline \multicolumn{5}{|c|}{ Criterios } \\
\hline Técnicos & Económicos & Sociales & Ambientales & Riesgo \\
\hline $\begin{array}{l}\text { 1. Madurez de la } \\
\text { tecnología (4.17) }\end{array}$ & $\begin{array}{l}\text { 1. Costos de operación } \\
\text { y mantenimiento (3.89) }\end{array}$ & $\begin{array}{l}\text { 1. Aceptación de la } \\
\text { comunidad (4.53) }\end{array}$ & $\begin{array}{l}\text { 1. Recursos } \\
\text { locales: agua y } \\
\text { suelo (4.55) }\end{array}$ & $\begin{array}{l}\text { 1. Política } \\
\text { energética (3.77) }\end{array}$ \\
\hline $\begin{array}{l}\text { 2. Eficiencia energética } \\
\text { (3.77) }\end{array}$ & $\begin{array}{l}\text { 2. Potencial de } \\
\text { recursos (3.83) }\end{array}$ & $\begin{array}{l}\text { 2. Generación de } \\
\text { empleos locales } \\
\text { (4.26) }\end{array}$ & $\begin{array}{l}\text { 2. Impacto en el } \\
\text { ecosistema (4.23) }\end{array}$ & $\begin{array}{l}\text { 2. Fenómenos } \\
\text { naturales (3.68) }\end{array}$ \\
\hline $\begin{array}{l}\text { 3. Disponibilidad de } \\
\text { repuestos y } \\
\text { mantenimiento (3.64) }\end{array}$ & $\begin{array}{l}\text { 3. Inversión inicial } \\
\text { (3.74) }\end{array}$ & $\begin{array}{l}\text { 3. Energía para } \\
\text { escuelas y centros } \\
\text { de salud (3.91) }\end{array}$ & 3. Emisiones de & 3. Fácil acceso \\
\cline { 1 - 3 } 4. Confiabilidad (3.53) & 4. Incentivos para la \\
\cline { 1 - 3 } $\begin{array}{l}\text { 5. Infraestructura física } \\
\text { (3.49) }\end{array}$ & $\begin{array}{l}\text { inversión (3.53) } \\
\text { territorios indígenas } \\
\text { (3.64) }\end{array}$ & gases (3.55) & $\begin{array}{l}\text { 4. Riesgos del } \\
\text { país (3.53) }\end{array}$ \\
\hline
\end{tabular}

Como era de esperar, tanto la cantidad como el alcance de los criterios tienen similitudes y diferencias con los proyectos referenciados en esta investigación. Dependiendo del lugar de aplicación, de los criterios inicialmente considerados, de los perfiles de los decisores y del criterio del autor; así serán los criterios definidos. En ese sentido, se puede referenciar el trabajo realizado por Parodi (2013), en su investigación para la evaluación de proyectos en el sector energético. Luego de la participación de expertos para definir los tipos de criterios a utilizar en los proyectos de planificación energética, pasó de contar con 44 criterios principales a 24 criterios distribuidos así: 6 técnicos, 2 económicos, 7 ambientales, 4 sociales, 3 estratégicos y 2 de riesgo. En la misma dirección, Rojas y Yusta (2015) en su trabajo de investigación en planificación energética, a partir de una revisión bibliográfica definieron 24 criterios, los cuales a partir de la experiencia del autor fueron reducidos a 13 criterios: 4 técnicos, 2 económicos, 4 ambientales y 3 sociales.

En el caso particular de los criterios técnicos, se pueden realizar comparaciones con el trabajo de Parodi (2013) quien inicialmente a partir de la revisión bibliográfica definió 8 criterios técnicos para someterlos a la valoración de expertos académicos en el área de energías renovables. Utilizando el promedio como referente para la selección, los criterios fueron reducidos a 5: madurez de la tecnología, flexibilidad de la tecnología, eficiencia energética, disponibilidad, infraestructura física y "Saber-Como" local. 
En relación a los criterios económicos, se puede referenciar el trabajo realizado por Kuthanazhi y Rao (2012) quienes a diferencia del presente trabajo realizaron un solo proceso de selección para los criterios económicos, basados en su experiencia y en la revisión bibliográfica de trabajos de investigación. De esta forma, sólo definieron 3 criterios para la planificación energética: costo actual, costos futuros y costos de transporte e instalación. Por otra parte, para los criterios sociales es de resaltar el trabajo realizado por Rojas y Yusta (2015) quienes en la etapa de identificación definieron 6 criterios sociales, a diferencia del presente trabajo realizaron un proceso de simplificación basado en la experiencia del autor para utilizar sólo 3 criterios: creación de empleos, aceptación social de la fuente de energía e índice de desarrollo humano.

Para los criterios ambientales, es de mencionar los resultados obtenidos por Demirtas (2013) a partir de la integración de criterios de diferentes trabajos de investigación relacionados con la planificación energética. Se definieron 2 criterios ambientales: impacto en el ecosistema y emisión de $\mathrm{CO}_{2}$; los cuales fueron definidos a partir de la experiencia del autor sin someterlos a juicio de expertos. Finalmente, en relación a los criterios de riesgo se puede referenciar el trabajo realizado por Aragonés et al. (2014) para seleccionar proyectos de energía fotovoltaica, en la que a diferencia del presente trabajo utilizaron 3 criterios de riesgo: cambio en políticas energéticas, vandalismo e inundación; los cuales se definieron a partir de una revisión bibliográfica y luego fueron sometidos a un proceso de priorización.

\section{CONCLUSIONES}

Los resultados presentados permiten concluir lo siguiente: 1) la aplicación del procedimiento propuesto permitió la participación de un grupo de expertos para seleccionar 20 subcriterios que pueden ser utilizados en proyectos de planificación energética en las zonas rurales de Colombia utilizando fuentes renovables de energía, 2) el baremo diseñado permitió medir el nivel de pertinencia de cada uno de los criterios y por lo tanto sirvió de soporte para el proceso de selección, 3) los subcriterios seleccionados permiten una evaluación integral de los proyectos de planificación energética teniendo en cuenta 5 criterios: técnicos, económicos, sociales, ambientales y riesgo, 4) los criterios económicos, sociales y ambientales tuvieron un nivel de relevancia Alto, mientras que los criterios técnicos y de riesgo tuvieron un nivel Moderado de relevancia, 5) la metodología propuesta permitió descartar 13 criterios que a juicio de los expertos no son pertinentes para los proyectos de planificación energética en las zonas rurales colombianas.

\section{AGRADECIMIENTOS}

Los autores agradecen al Fondo Patrimonial para la Investigación (Fonciencias) de la Universidad del Magdalena por la financiación del presente proyecto.

\section{REFERENCIAS}

Ahmad, S. y Tahar, R., Selection of renewable energy sources for sustainable development of electricity generation system using analytic hierarchy process: A case of Malaysia, Renew Energ, 63, 458-466 (2014)

Amer, M. y Daim, T., Selection of renewable energy technologies for a developing county: A case of Pakistan, Energy Sustain Dev, 15(4), 420-435 (2011)

Aragonés, P., Chaparro, F., Pastor, J. y Pla, A., An AHP (Analytic Hierarchy Process)/ANP (Analytic Network Process)based multi-criteria decision approach for the selection of solar-thermal power plant investment projects, Energy, 66, 222$238(2014)$

Baris, K. y Kucukali, S., Availability of renewable energy sources in Turkey: Current situation, potential, government policies and the EU perspective, Energ Policy, 42, 377-391 (2012)

Corpoema, Formulación de un plan de desarrollo para las fuentes no convencionales de energía en Colombia. Volumen 2 - Diagnóstico de las FNCE en Colombia, (en la web: https://goo.gl/rqA1jV, acceso: 15 de agosto de 2017), UPME (2010)

De la Hoz, E., González, Á. y Santana, A., Metodología de Medición del Potencial Exportador de las Organizaciones Empresariales, doi: 10.4067/S0718-07642016000600003, Inf. Tecnol. 27(6), 11-18 (2016)

Demirtas, O., Evaluating the Best Renewable Energy Technology for Sustainable Energy Planning, IJEEP, 3, 23-33 (2013)

Emodi, N., Shagdarsuren, G. y Tiky, A., Influencing Factors Promoting Technological Innovation in Renewable Energy, IJEEP, 5(3), 889-900 (2015)

Espejo, C., García, R. y Aparicio, A., El resurgimiento de la energía minihidráulica en España y su situación actual, Rev Geogr Norte Gd, (67), 115-143 (2017)

Garcia, R. y Zerda, A., Hydropower Megaprojects in Colombia and the Influence of Local Communities: A View from Prospect Theory to Decision Making Process based on Expert Judgment used in Large Organizations, IJEEP, 6(3), 408420 (2016)

Gosavi, A., Analyzing Responses from Likert Surveys and Risk-adjusted Ranking: A Data Analytics Perspective, Procedia Comput Sci, 61, 24-31 (2015) 
Guerrero, G., Sánchez, J., García, M., Lamata, M. y Verdegay, J., Decision-Making for Risk Management in Sustainable Renewable Energy Facilities: A Case Study in the Dominican Republic, Sustainability, 8(5), 1-21 (2016)

Gülçin, B. y Sezin, G., A new GDM based AHP framework with linguistic interval fuzzy preference relations for renewable energy planning, J Intell Fuzzy Syst, 27, 3181-3195 (2014)

Hernández, R., Fernández, C., Baptista, M., Metodología de la investigación, 5a edición, 300-303. Mc Graw Hill, México D.F, México, (2010)

Hernández, D., Urdaneta, A. y De Oliveira, P., A hierarchical methodology for the integral net energy design of small-scale hybrid renewable energy systems, Renew Sust Energ Rev, 52, 100-110 (2015)

IRENA, Renewables 2016: Global Status Report (en la web: https://goo.gl/9UwJf7, acceso: 29 de agosto de 2017), IRENA (2016)

Kahraman, C., Kaya, I. y Cebi, S., A comparative analysis for multiattribute selection among renewable energy alternatives using fuzzy axiomatic design and fuzzy analytic hierarchy process, Energy, 34, 1603-1616 (2009)

Kang, H., Hung, M., Pearn, W., Lee, A. y Kang, M., An Integrated Multi-Criteria Decision Making Model for Evaluating Wind Farm Performance, Energies, 4, 2002-2026 (2011)

Kumar, A., Deng, Y., He, X., y Kumar, P., A Multi Criteria Decision based rural electrification system, IECON 2016 - 42 nd Annual Conference of the IEEE Industrial Electronics Society, 4025-4030, Florence-Italy, 23 to 26 October (2016)

Kuthanazhi, V. y Rao, A., Selection of Photovoltaic Modules for Off-Grid Rural Application Based on Analytical Hierarchy Process (AHP), 38th IEEE Photovoltaic Specialists Conference IEEE, 2888-2893, Austin-USA, 3 to 8 June (2012)

Mizanur, M., Paatero, J. y Lahdelma, R., Evaluation of choices for sustainable rural electrification in developing countries: A multicriteria approach, Energ Policy, 59, 589-599 (2013)

Mourmouris, J. y Potolias, C., A multi-criteria methodology for energy planning and developing renewable energy sources at a regional level: A case study Thassos, Greece, Energ Policy, 52, 522-530 (2013)

Parodi, V., Propuesta metodológica para la evaluación integral de proyectos en el sector energético, Tesis doctoral, Universidad Politécnica de Valencia, Valencia, España (2013)

Robles, C., Polo, A., Ospino, A., An AHP Based Approach for Evaluating Renewable Energy Sources, International Journal of Energy Economics and Policy, 7, 38-47 (2017)

Rojas, J. y Yusta, J., Application of multicriteria decision methods for electric supply planning in rural and remote areas, Renew Sust Energ Rev, 52, 557-571 (2015)

Rosso, M., Bottero, M., Pomarico, S., La Ferlita, S. y Comino, E., Integrating multicriteria evaluation and stakeholders analysis for assessing hydropower projects, Energ Policy, 67, 870-881 (2014)

Şengül, Ü., Eren, M., Eslamian, S., Gezder, V. y Bilal, A., Fuzzy TOPSIS method for ranking renewable energy supply systems in Turkey, Renew Energ, 75, 617-625 (2015)

Shabbar, S., Janajreh, I. y Ghenai, C., Sustainability index approach as a selection criteria for energy storage system of an intermittent renewable energy source, Appl Energ, 136, 909-920 (2014)

Simanca, M., Montoya, L. y Bernal, C., Gestión del conocimiento en cadenas productivas: El caso de la cadena láctea en Colombia, doi: 10.4067/S0718-07642016000300009, Inf. Tecnol. 27(3), 93-106 (2016)

Tasri, A. y Susilawati, A., Selection among renewable energy alternatives based on a fuzzy analytic hierarchy process in Indonesia, Sustainable Energy Technol Assess, 7, 34-44 (2014)

UPME, Integración de las energías renovables no convencionales en Colombia (en la web: https://goo.gl/QbXL9i, acceso 25 de agosto de 2017), UPME (2015)

Zanuttigh, B., Angelelli, E., Kortenhaus, A., Koca, K., Krontira, Y. y Koundouri, P., A methodology for multi-criteria design of multi-use offshore platforms for marine renewable energy harvesting, Renew Energ, 85, 1271-1289 (2016)

Zhang, Y., Xu, Q. y Sun, M., Perspectives for Utilization of Multicriteria Decision Methods AHP / ANP to Create a National Energy Strategy in Terms of Sustainable Development, Adv Mat Res, 616, 1585-1590 (2012) 